\title{
Un abordaje a las propuestas y proyectos de creación del túnel subfluvial Paraná-Santa Fe
}

Maximiliano Camarda ${ }^{1}$

\section{RESUMEN}

Desde principios del siglo XX se realizaron propuestas para la conexión de Entre Ríos, más de cincuenta años después se concretó. El objetivo de este trabajo es profundizar en el derrotero de las propuestas y proyectos sobre la creación de un túnel o puente.

\section{Abordagem das propostas e projetos para a criação do túnel subfluvial Paraná-Santa Fé RESUMO}

Desde o início do século XX, foram feitas propostas para a conexão de Entre Rios, mais de cinquenta anos depois foi finalizado. O objetivo deste trabalho é aprofundar o curso das propostas e projetos sobre a criação de um túnel ou ponte.

\section{INTRODUCCIÓN}

Desde principios del siglo XX se fueron presentadas en las Camaras de Senadores y Diputados de la Nación Argentina la creación de un túnel o puente que conecte la Mesopotamia con el resto del territorio nacional. Con la llegada del peronismo, las propuestas se transformaron en proyectos concretos. Una de los cuales fue el realizado por los ingenieros Carlos Laucher, Norberto Alagef y Corrado Vittori quienes en febrero de 1955 lo presentaron. El objetivo del proyecto era demostrar la factibilidad técnica- económica de la construcción y explotación de un túnel subfluvial entre las ciudades de Paraná y Santa Fe. Este proyecto fue el que, algunos años después se llevó a cabo. Este trabajo tiene como objetivo dar cuenta del proceso que se recorrió desde la primera propuesta hasta su concreción.

En este largo peregrinaje que duró casi 60 años desde la presentación de la primera propuesta hasta la puesta en funcionamiento del túnel, se desarrolló un sistema de conexión por medio de ferry boats y luego de balsas, que con el correr del tiempo y el incremento de la demanda, se fue sofisticando y ampliando. En los últimos años, los trabajos que se realizaron en torno al puente subfluvial estuvieron orientados en profundizar en las

\footnotetext{
${ }^{1}$ Doutor en Historia (Universidad Nacional de La Plata). Becario posdoctoral de CONICET y docente FCECO, Universidad Nacional de Entre Rios (Argentina). Email: maximilianocamarda@fceco.uner.edu.ar
} 
causas políticas de la concreción de este proyecto (De Marco, 2016) y en el ciclo de las políticas públicas que culminó con su ejecución (Mateo, Camarda, 2018).

\section{CONTEXTO REGIONAL}

Entre Ríos a fines del siglo XIX ocupaba un lugar central en el entramado económico y poblacional de la Argentina, alcanzando un tercer lugar, detrás de Buenos Aires y Santa Fe en torno a los ingresos generados en per cápita 1889; en 1937 ocupaba el séptimo lugar provincial (Rofman, Romero; 1990). Este proceso decadente, como veremos, continuó en las décadas siguientes, hasta la de 1960, en donde cambió ligeramente la tendencia.

Como se ha sostenido reiteradamente, la población de la Argentina durante la primera mitad del siglo XX se multiplicó exponencialmente, mientras que la población de Entre Ríos sufrió un proceso de estancamiento. En este sentido, se puede sostener que el movimiento poblacional provincial se puede dividir en tres etapas, uno de poblamiento vertiginoso entre 1869-1947, seguido de otro en el cual hay estancamiento y/o despoblamiento entre 1947 y 1980, para retomar un crecimiento moderado entre ese año y 2010 (Mateo, Rodríguez, Camarda; 2017)

Las causas de estas transformaciones están dadas, como en otras provincias argentinas, en una migración interna tras la fuerte inmigración europea de fines del siglo XIX e inicios del siglo XX. Este movimiento de población marcó durante las primeras décadas del siglo pasado la distribución territorial de la población rural-urbana, y de forma creciente, a partir de los años cuarenta la interurbana, en particular hacia el Área Metropolitana de Buenos Aires y las ciudades de Córdoba, Rosario, Mendoza, Tucumán y otras ciudades capitales de provincia.

Era un proceso emergente del de industrialización por sustitución de importaciones, que redundaba en ganancias de productividad al trasladarse fuerza de trabajo de ramas de baja a alta productividad (Busso, 2007) Como sostiene Biasizo (2015), el desarrollo de la Industrialización por Sustitución de Importaciones no tuvo impacto en Entre Ríos, pero tampoco hubo una expansión en cuanto a las actividades agropecuarias.

La dinámica poblacional tuvo su correlato en la producción agropecuaria, la cual mantuvo un proceso de estancamiento pronunciado durante la primera mitad del siglo XX. Con respecto a las distintas producciones agropecuarias, siguiendo lo observado por Rofman y Romero (1990) para la distribución del PBI en las regiones y lo desarrollado de la 
población, en este sentido también tiene menor influencia. El desarrollo de la ganadería, que había sido central durante el siglo XIX, en el siglo XX tuvo un estancamiento muy marcado. La ganadería vacuna paso a la mitad a mediados de la década de 1910 de la que existía en 1888 y se mantuvo hasta la década de 1990 en los mismos parámetros. Si bien, a principios del siglo XX, se puede observan un reemplazo con el ganado ovino, para la década de 1920 decaen todas las producciones ganaderas.

En cuanto a la producción agraria se puede sostener que, a diferencia de la ganadería que estuvo un siglo XX de estancamiento, que progresivamente se incrementaron las cantidades de hectáreas cultivadas. Como ejemplo de ello, tomamos cuatro cultivos: trigo, maíz, lino y avena y si observamos salvo el caso del trigo (que a fines del siglo XIX era uno de los pocos cultivos y por ello predominante) las otras tres producciones fueron incrementado el territorio cultivado. En particular el lino y la avena tuvieron un desarrollo sustantivo. En este sentido, quedará para futuros trabajos establecer la relación de la pérdida de población a nivel regional y el incremento de las superficies sembradas (que requieren mayor mano de obra que la ganadería) siendo probable en este sentido que gran parte de la población que migro en la década de 1940 se encontrara afincados en las ciudades y pueblos y no en el campo.

\section{PRIMERAS PROPUESTAS}

El 29 de mayo de 1908 luego de idas y venidas y de instalaciones en lugares anegadizos en temporadas de lluvias, se inauguró el primer ferry boat. Conectaba Zárate con Ibicuy y para su inauguración se encontraba el Presidente de la Nación Dr. Figueroa Alcorta, el Ministro de Obras Públicas Dr. Ezequiel Ramos Mejía, los Ministros de Hacienda, Agricultura y Guerra, el Gobernador de la Provincia de Buenos Aires Ignacio Irigoyen, el Intendente de Bs. As. Manuel Güiraldes, Ministros de Inglaterra, Francia entre otras personalidades de renombre.

Unos años más tarde, en 1911, en la Cámara de Senadores de la Nación, los senadores Láinez, Virasoro, Maciá, Carbó, Terán, Resoagli y Obejero presentaron un proyecto para la realización de una conexión ferroviaria del puerto de Ibicuy con la Provincia de Buenos Aires. En 1918, el Senador Méndez Casariego lo retomó este proyecto y logró que el 7 de marzo de ese año se iniciaran trabajos de estudio sobre la factibilidad de realizarlo. 
Los resultados de este proyecto no fueron favorables, pese a ello, dada la necesidad de vinculación, se le sumaron al ferry primogénito dos nuevos. Unos años mas tarde, entre 1926 y 1929 tres barcos más, estos últimos unían Ibicuy con la desembocadura del Riachuelo en Buenos Aires. ¿Cómo era el sistema? El mecanismo era el siguiente: Los barcos debían acercarse hasta quedar encastrados en un muelle de madera, se juntaban los rieles y los trenes debían subir y bajar en ese atracadero. El puerto de Ibicuy era utilizado como centro de abastecimiento y reparación ya que contenía los servicios de mantenimiento, administración y proveeduría (Cusmai, 2014)

Si bien los accidentes no eran usuales, cuando se producía uno, generaba grandes pérdidas materiales y retrasaba el desarrollo del servicio durante tiempos prolongados. El 30 de Junio de 1926, a las 9.30 de la mañana, se produjo un choque entre dos formaciones: el Carbó y el Parera, lo que produjo graves daños en la primera formación y el hundimiento de la segunda. El Carbó transportaba 24 vagones jaulas con ganado y el Parera ocho vagones también de carga. A partir del accidente durante el resto de 1926 se utilizó solo el único barco que no participo de la colisión, el Lacroze, haciendo mucho más dificultoso el transporte de personas y de carga.

Dos semanas después del accidente, los Senadores Melo, Etchevehere y Vidal presentaron en el Senado de la Nación un proyecto para la realización de un túnel en Ibicuy. Enlazó dos argumentos para dar cuenta de los beneficios de su realización, el primero giraba en torno a que sería “...una obra cada vez más exigida por el progreso de las regiones que ha de vincular, pues está de manifiesto el vigor con que Corrientes, Entre Rios y Misiones están lanzados a la agitación de su industrialismo agrario, como está de manifiesto también, la necesidad de ofrecer a Paraguay un medio de transporte rápido, seguro y orgánico, propicio a un intercambio de beneficios recíprocos..." (El diario, Paraná, viernes 23 de Julio de 1926) A continuación da cuenta de la precariedad del sistema de ferry boat a partir del accidente y también de las dificultades que ese sistema producía.

A partir de la presentación de la propuesta se realizaron, por parte del Gobierno Nacional, dos medidas concretas: se incorporaron dos nuevas formaciones de barcos para el traslado de los ferrocarriles y en diciembre del mismo año se inauguró el servicio de lanchas para el traslado de pasajeros entre Paraná y Santa Fe. Finalmente, al año siguiente se anunció la construcción de balsas para el transporte de vehículos realizando el trayecto Paraná- Santa Fe, para ello se construyeron además embarcaderos para ese fin en las dos márgenes del río. 
Este servicio se inauguró el 27 de febrero de 1929, se realizaban dos viajes diarios y se transportaba un máximo de 400 personas y 100 vehículos por día.

Para el año 1931 se encontraban dos balsas en servicio realizando 8 viajes diarios y 3267 viajes en el año. El impacto de este servicio fue muy importante ya que se transportaron más de 15.000 automóviles, de 20.000 camiones y 210.000 pasajeros, logrando una recaudación de más de 330.000 pesos, en el cuadro siguiente se puede observar el desmenuzamiento de los datos.

Este sistema de balsas logró absorber la demanda de transporte para el cruce del rio, siendo ampliado a otros, en 1935 se inauguró el servicio Victoria- Rosario y en 1937 Zarate y Constanza. El incremento durante la década de 1930 del movimiento de personas, automóviles y camiones fue en crecimiento pese al estancamiento de los primeros años producto de la crisis internacional.

En este contexto de expansión del servicio de las balsas fue presentada una nueva propuesta. En 1936 por el senador Atanasio Eguiguren por el que sea aprobada la realización de un túnel que comunicase Paraná con Santa Fe. Como sostiene Miguel Ángel de Marco (h) (2016) este proyecto fue el antecedente más ambicioso, completo y costoso de los que en adelante se proyectarían. El proyecto constaba de seis artículos, en donde plantea la conformación de una comisión especial para realizar los estudios, proyectos y presupuestos, la obra se ejecutaría no solo para que sea de paso de automóviles y camiones sino también asegurar la circulación de los ferrocarriles y el ejecutivo debía adelantar $\$ 120.000 \mathrm{~m} / \mathrm{n}$ para el inicio de los trabajos.

El argumento central de Eguiguren para la realización del túnel es que si bien las balsas habían logrado un desarrollo importantísimo, no era la solución definitiva. En particular, la mayor complicación la encuentra en el tiempo de demora que representa el viaje, Eguiguren en la argumentación en la Cámara de Senadores aclara:

Un ejemplo al caso: Un pasajero sale de Retiro a las 20 horas y llega a Santa Fe a las 6 del día siguiente. Para ir en la primera balsa a Paraná debe esperar hasta las 9, para llegar más o menos a las 11. Los 500 kilómetros, aproximados entre Buenos Aires y Santa Fe, le insumen 10 horas; los 27 kilómetros de Santa Fe a Paraná, fíjense señores senadores, se cubren en 5 horas, amén de los inconvenientes del transbordo y del aumento de gastos consiguientes, desembarcar y viaje de la estación al puerto, y embarque y desembarque en Paraná. De existir el túnel o el puente, saliendo de buenos Aires a las 20 horas, se podría estar en Paraná a las 6:15 del día siguiente; hoy se llega, como he dicho, por la balsa, a las 11 horas. (De Marco, 2016: 37) 
El proyecto continuó un curso lento pero nunca fue rechazado, como sostiene el mismo Aguiguren tiempo después:

\begin{abstract}
Mi proyecto de puente o túnel entre Paraná y Santa Fe, nunca fue archivado, sino tratado y resuelto por el Congreso de la Nación, pruebas: $1^{\circ}$ En la ley de presupuestos de 1939, conseguí incorporar una partida de $\$ 120.000$ para los estudios preliminares, $2^{\circ}$ por la Ley Nacional $\mathrm{N}^{\circ} 12.576$ y 12.595 , conseguí incorporar una partida de $\$ 10.000 .000,3^{\circ}$ como llegamos al año 1941[...] Presente junto a mi compañero de banca, senador Eduardo Laurencena, una minuta de comunicación al poder ejecutivo para que haga los estudios para unir las ciudades de Paraná y Santa Fe, mediante puente o túnel [...] (El Diario, 16/12/1969)
\end{abstract}

Como se puede apreciar, se mantuvo la creación de la obra pública presente pero no se avanzó lo suficiente como para una ejecución al corto plazo. Entre las causas de su no desarrollo encontramos como central, que la iniciativa tenga como punto fuerte el tiempo de demora de las balsas ya que, si bien era mucho más lenta, la inversión que debía realizar la nación para la creación de un túnel era muy importante. Por otro lado, el crecimiento de la dinámica durante la década de 1930 no continuó durante la década siguiente.

A partir de la caída del tráfico por balsas, la creación del túnel volvió a la oscuridad durante un tiempo, hasta que se produjo la reactivación durante la década de 1950 siendo esto un motivo de relanzamiento de la propuesta del túnel.

\title{
4 PROYECTO DEL PERONISMO
}

En el marco del segundo plan quinquenal, se desarrolló un informe realizado por los ingenieros Carlos Laucher, Norberto Alagef y Corrado Vittori quienes en febrero de 1955 lo presentaron. El objetivo del proyecto era demostrar la factibilidad técnica- económica de la construcción y explotación de un túnel subfluvial entre las ciudades de Paraná y Santa Fe. Anclan su argumento en los capítulos XXIII y XXIV del segundo plan quinquenal en donde se expresa dentro de los objetivos: " $1{ }^{\circ}$ Facilitar la vinculación entre núcleos poblados del país, $2^{\circ}$ Asegurar la prestación de servicios con regularidad, $3^{\circ}$ Propender al desarrollo y fomento de las economías regionales, $4^{\circ}$ Abaratar el transporte, $5^{\circ}$ Contribuir a la promoción del turismo nacional e internacional y $6^{\circ}$ Las previsiones de la defensa nacional." 


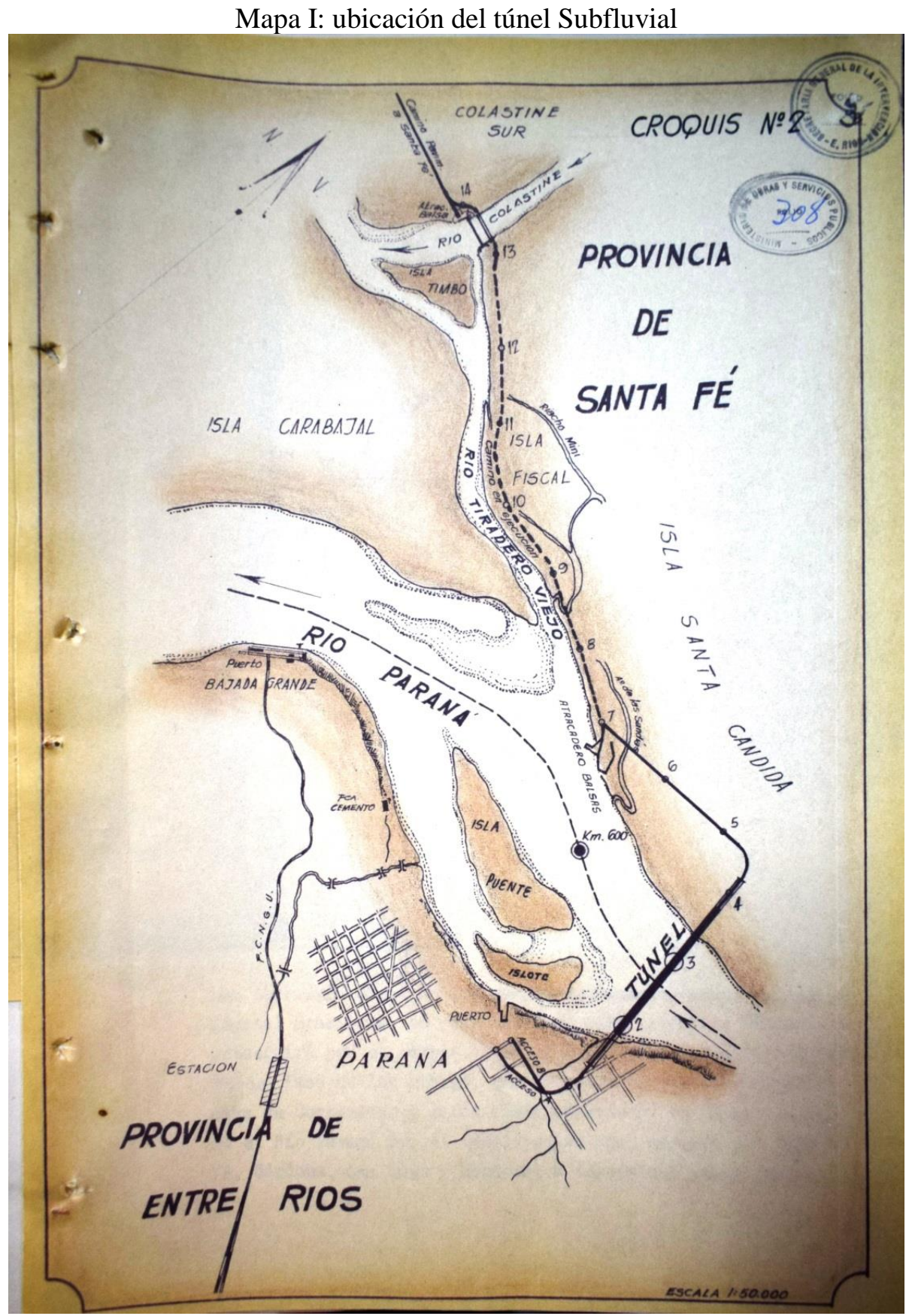

Fuente: Ministerio de Obra Pública. Expediente 61208. AGER

En 1954 se publicó un informe en donde se analiza el "aislamiento" de Entre Ríos con el resto del país, el proyecto de concreción, el impacto económico y el estudio técnico.

En primer lugar, el análisis plantea las dificultades históricas que sufrió Entre Ríos a causa de su aislamiento y dado que es un territorio cuya producción fundamental era agropecuaria requeriría una mayor velocidad de llegada a los destinos de esos productos que los ofrecidos por el transporte fluvial. Hace hincapié, sin ofrecer datos sobre ello, que en Entre Ríos había una producción de productos de granja (huevos, aves, frutales, etc) que no era posible transportar por su característica perecedera. En la misma línea, realiza un recorrido 
sobre las transformaciones en el transporte, en particular los ferry-boats y las balsas. Establece que la salida de cabezas antes de la implementación de los Ferry-boats era de 40.000 anuales y una vez puesto en funcionamiento el servicio paso a 100.000. La implementación del sistema de balsas, observa, mejoró notablemente la comunicación, en particular el transporte de mercancías, vehículos y pasajeros, pese a ello sostiene que para la década de 1950 persiste la falta de comunicación que genera una serie de problemas económicos, sociales, políticos y militares. Cómo solucionar esta problemática?

\begin{abstract}
El túnel proyectado, es la única solución técnica-económica ya que la construcción de un puente exige una obra monumental para permitir la libre navegación en el Paraná. Un puente colgante para unir Paraná con Santa Fe requiere una inversión de aproximadamente 2.000 millones de pesos, seis veces superior al coste del túnel. Además, se plantearía un problema de divisas, ya que sería necesario importar unas 100.000 toneladas de aceros especiales.
\end{abstract}

Además, sostienen que para su realización sería necesaria una confluencia de capitales estatales y privados. Tomando los datos de 1952 y los anteriores, en donde se apunta la cantidad de vehículos, 107.000 y pasajeros 446.000, los ingenieros realizaron una proyección de los incrementos en la circulación de los siguientes 50 años y calcularon la tarifa en torno a ello. Estimaron que el plazo mínimo de la concesión sería de 42 años, por lo cual plantean que por las características de la obra y asegurarle al capital privado un margen de seguridad el plazo de la concesión sería ser de 50 años.

A principios de la década de 1950 se duplicó la cantidad de vehículos que circularon por las balsas, en el proyecto se plantea la necesidad de realizar un cambio estructural dado que el sistema de balsas generaba un retraso muy importante en el transporte, pese a ello no se llevó a cabo y no fue tratado. Probablemente el peronismo, producto de que aún la demanda por parte de los vehículos transportados no eran los suficientes para garantizar el éxito de una obra de la envergadura del túnel, opto por el fortalecimiento de las balsas a partir de la creación en 1949 de la "Flota Argentina de Navegación Fluvial".

Si volvemos a ver el último gráfico, se puede observar que al incremento que señalan los ingenieros a principios de la década de 1950 fue duplicada una década después y hacia fines de la década de 1960 se triplican los 100.000 vehículos que eran transportados por las balsas en el trayecto Paraná- Santa Fe.

El proyecto realizado durante el peronismo, continuó su curso tras el golpe, el anteproyecto fue publicado en 1956 en la Revista del Centro de Ingenieros de la Provincia de Buenos Aires, al año siguiente se emitió un Decreto le que autorizaba al Ministerio de Obras 
Publicas la realización del túnel. En la nota que presentaron los ingenieros que luego se incluyó en el decreto se destaca la importancia del túnel para la vinculación interoceánica anexando un mapa del circuito. Plantea que:

\begin{abstract}
"La sola consideración de dos puntos fundamentales darán a V. E. la dimensión de lo que la obra habrá de representar en el futuro para el progreso de la Mesopotamia Argentina y de las vinculaciones a que dará lugar en el orden Internacional. Con respecto a esto +ultimo, debo expresar que el túnel será el nexo que mediante la gran carretera panamericana transversal que parte de Río de Janeiro y llega a Valparaiso en Chile, establecerá el vínculo interoceánico del Atlántico con el Pacífico". Ministerio de Obra Pública. Expediente 61208. AGER. Página 3.
\end{abstract}

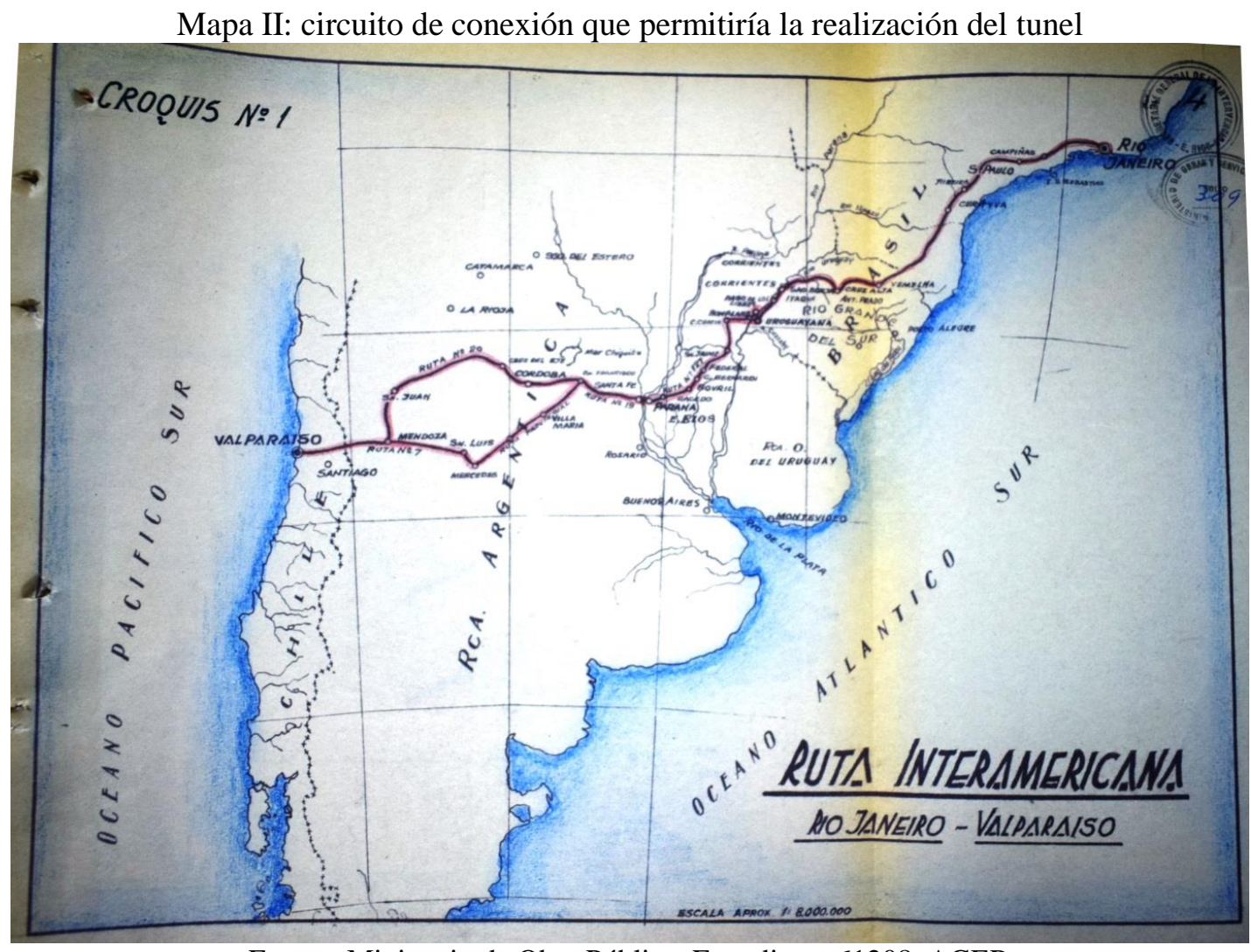

Fuente: Ministerio de Obra Pública. Expediente 61208. AGER

Durante el frondizismo continuó su recorrido articulando la política nacional con las de Entre Ríos y Santa Fe, en particular a partir de que el 15 de junio de 1960, en Santa Fe, firmaron los Gobernadores Sylvestre Begnis y Uranga el tratado interprovincial que dio inicio un tiempo después la ejecución de la obra. El gobierno nacional, entre tanto intentaba continuar apostando por el incremento de la circulación de balsas, mecanismo que le era favorable porque no debía desembolsar dinero para su construcción e incrementaba su ingreso fiscal a partir del cobro de los cruces de vehículos. Dado ese contexto, las provincias buscaron un mecanismo de financiación interna y fue decisivo para adoptar la opción túnel debido a 
que, según interpretación legal, bajo el lecho del río se extendían las jurisdicciones provinciales y cesaba la jurisdicción nacional (Mateo, Camarda 2018).

Como sostiene Berrotarán y Pereyra (2012), en este caso se puede apreciar que, si bien las ideas en torno a la creación de un túnel vienen de vieja data, el proyecto de concreción de este fue llevado a cabo durante el peronismo. Las historiadoras sostienen que: "En la década de 1930 el estado amplió el campo de sus actividades, replicando esquemas organizacionales existentes, y es en el peronismo clásico cuando los cambios institucionales condujeron a un replanteo en el plano organizativo" (Berrotarán y Pereyra, 2012: 145)

\section{CONCLUSIÓN}

Desde principios del siglo XX se realizaron en distintos momentos presentaciones sobre la necesidad de establecer un vínculo permanente que conecte las ciudades de Santa $\mathrm{Fe}$ y Paraná. A nuestro entender, las presentaciones previas al proyecto del peronismo, tuvieron como objetivo el desarrollo y/o ampliar los mecanismos de conexión no permanente, ferry boats y balsas, dado que no se encontraban las condiciones económicas para realizar una obra de infraestructura de gran escala.

Las proyecciones realizadas durante el peronismo, demuestran que el crecimiento de los vínculos a partir del incremento del número de vehículos que generaban grandes colas para la utilización de las balsas, hacían obsoleto ese mecanismo. Por ese motivo, durante el peronismo se realizó un proyecto de concreción de un túnel (se descarta la realización de un puente por su costo). En las décadas siguientes se concreta las estimaciones, con lo cual, la realización de un túnel era doblemente beneficioso, dado que permitía la conexión concreta de los territorios y, dada la masividad, financiar su coste.

La proyección que realizaron tenía como fin conectar por tierra a Entre Ríos y permitir la vinculación interoceánica de un circuito que conectaría Río de Janeiro con Valparaíso.

\section{REFERÊNCIAS}

BALLENT, Anahí (2008) Ingeniería y Estado: la red nacional de caminos y las obras públicas en la Argentina, 1930-1943.História, Ciências, Saúde - Manguinhos, Rio de Janeiro, v.15, n.3, p.827-847, jul.-set. 2008. 
BERROTARÁN, P. y PEREYRA, E. (2012). Momentos y procesos para una historia del Estado en Argentina. Aportes para el Estado y la administración gubernamental, (30), pp. 141-167.

BIASIZO, Rogelio José (2015) Economía de Entre Ríos en el período de intervencionismo conservador (1930 1945). Paraná. EDUNER.

BUSSO, Gustavo (2007). Migración interna y desarrollo territorial en Argentina a inicios del Siglo XXI. Brechas e impactos sociodemográficos de la migración interna interprovincial. IX Jornadas Argentinas de Estudios de Población. Asociación de Estudios de Población de la Argentina, Huerta Grande, Córdoba.

DE MARCO, Miguel Angel (2016) El túnel subfluvial. Federalismo y desarrollo. Santa Fe. Ediciones UNL.

GÓMEZ, Teresita y Silvia Tchordonkian (2015) "Debates en torno a la construcción de un sistema vial en Argentina y su concreción en la acción de gobierno entre 1940 y 1950." Documento de trabajo. Nro. 45 Centro de Estudios de la Situación y Perspectivas de la Argentina. http://www.economicas.uba.ar/wp-content/uploads/2015/11/Doc-de-trabajo-N45 1.pdf.

GARCIA HERAS, Raúl (1990): "Las compañías ferroviarias británicas y el control de cambios en la Argentina durante la Gran Depresión", en revista Desarrollo Económico Vol. 29 n$^{\circ} 116$, enero-marzo 1990.

MATEO, Jose y Maximiliano CAMARDA (2016). Un puente hasta el túnel entre Santa Fe y Paraná. Los intentos de conexión carretera de la mesopotamia argentina hasta su instalación definitiva en la agenda de las políticas públicas (1911-1960). En A. A. Nieto \& G. Yurkievich (Eds.), Los Espacios portuarios. Mar del Plata: Agencia Nacional de Promoción Científica.

ORTEGA, Fernado (2005) "La influencia ferroviaria en el desarrollo económico de la Mesopotamia (1874-1906)". En: X Jornadas Interescuelas/Departamentos de Historia. Escuela de Historia de la Facultad de Humanidades y Artes, Universidad Nacional del Rosario. Departamento de Historia de la Facultad de Ciencias de la Educación, Universidad Nacional del Litoral, Rosario.

ORTIZ, R. (1943). Valor económico de los puertos argentinos. Buenos Aires: Losada.

ORTÍZ, R. (1987). Historia económica de la Argentina. Buenos Aires: Plus Ultra.

OSPITAL, María Silvia (2005) Turismo y territorio nacional en Argentina. Actores sociales y políticas públicas, 1920-1940. E.I.A.L., V. 16 N 2

RASCOVAN, Alejandro. Las empresas ferroviarias en las provincias de Entre Ríos, Corrientes y Misiones (1866-2014). Entre intereses globales, nacionales y regionales: Revista Transporte y Territorio, N 13. 